\title{
A utilização da Goal Programming no processo de tomada de decisão
}

Doutorado em Controladoria e Contabilidade pela Faculdade de Economia e Administração de São Paulo - FEA/USP Professora da Universidade de São Paulo - FEARP/USP Avenida Bandeirantes, 3900. Ribeirão Preto/SP. CEP: 14040-900 E-mail: flaviazd@usp.br

Dimitri Pinheiro de Sant'Anna Mestrado em Ciências Contábeis pela Fundação Instituto Capixaba de Pesquisas Contabilidade Economia e Finanças - FUCAPE Avenida Fernando Ferrari, 1358. Goiabeiras. Vitória/ES. CEP: 29075-010 E-mail:dimitri_ps@yahoo.com.br

Luciene Laurett Rangel Mestrado em Ciências Contábeis pela Fundação Instituto Capixaba de Pesquisas Contabilidade Economia e Finanças - FUCAPE Avenida Fernando Ferrari, 1358. Goiabeiras. Vitória/ES. CEP: 29075-010 E-mail: luciene.rangel@br.nestle.com

Valcemiro Nossa Doutorado em Controladoria e Contabilidade pela Faculdade de Economia e Administração de São Paulo - FEA/USP Professor da Fundação Instituto Capixaba de Pesquisas Contabilidade Economia e Finanças - FUCAPE Avenida Fernando Ferrari, 1358, Goiabeiras. Vitória/ES. CEP: 29075-010 E-mail:valcemiro@fucape.br

\section{RESUMO}

O objetivo deste artigo é demonstrar como o gestor pode aplicar a técnica do goal programming na formulação de alternativas de um plano operacional. Esta técnica requer um procedimento de solução iterativa, pela qual o tomador de decisão investiga uma variedade de soluções para encontrar a que seja mais satisfatória. Para tanto, por meio de um exemplo prático, demonstra-se a operacionalização dessa técnica, com a utilização do recurso "solver", disponível na planilha eletrônica Microsoft Excel. O modelo matemático do goal programming (programação por metas), por meio de simulações, permite que os gestores, durante o processo de planejamento ou de reestruturação da empresa, possam estar, continuamente, revendo suas prioridades e a própria hierarquia das metas/objetivos da empresa. Portanto, este trabalho visa contribuir para a maior utilização dos modelos matemáticos e estatísticos no cotidiano empresarial, buscando melhorar o processo de tomada de decisão. A utilização de 
A utilização da Goal Programming no processo de tomada de decisão Flávia Zobóli Dalmácio, Dimitri Pinheiro de Sant’Anna, Luciene Laurett Rangel, Valcemiro Nossa

modelos facilita a compreensão de estruturas e relações complexas, pois segrega os aspectos fundamentais relativos ao problema em foco.

Palavras-chave: Goal programming. Modelo matemático. Iterações. Metas. Planejamento. Gestão.

\section{The use of Goal Programming in decision process}

\section{ABSTRACT}

The purpose of this article is to demonstrate how the manager can apply the technique of goal programming in the formulation of alternatives of an operational plan. This technique requires an iterative solution process, whereby the manager investigates a variety of solutions to find one that is more satisfactory. Through a practical example, we show the operationalization of this technique, using the feature "solver", available in Microsoft Excel spreadsheet. The mathematical model of the goal programming (programming by goals), through simulations, enables that managers during the process of planning or restructuring of the company, may be, continuously, reviewing them priorities and the hierarchy of goals / objectives of the company. So, this paper, aims at contributing to the increased use of mathematical and statistical models, in the daily business, seeking to improve the decision process. The use of models facilitates understanding of complex structures and relationships, because it separates the fundamental aspects of the problem into focus.

Key-words: Goal programming. Mathematical model. Iterations. Goals. Planning. Management.

\section{INTRODUÇÃO}

Para definir os rumos e os resultados de uma empresa, os gestores dependem de ferramentas que os auxiliem no processo de tomada de decisão. Uma decisão, segundo Andrade (2000, p. 2), "é um curso de ação escolhido pela pessoa, como meio mais efetivo à sua disposição para alcançar os objetivos procurados, ou seja, para resolver o problema que a incomoda".

De acordo com Arantes (1998, p. 130), "o subsistema gerencial fornece os instrumentos de 'inteligência' para a administração criar o futuro desejado e assegurar que as ações estão no caminho correto para realizar esse futuro". 
O planejamento é um dos processos do subsistema gerencial utilizado para definir os caminhos que devem ser seguidos para se atingir o futuro desejado. Esse processo é composto por várias fases: definição dos resultados, avaliação das condições, formulação de alternativas, avaliação, escolha e detalhamento do plano (ARANTES, 1998, p. 138).

Definidos os resultados e avaliadas as condições, internas e externas, favoráveis e desfavoráveis da empresa, o gestor, na fase de formulação de alternativas para a elaboração do plano operacional, pode contar com técnicas da pesquisa operacional, tais como: programação linear, teoria dos jogos, teoria das filas, programação dinâmica, análise de risco, goal programming (programação por metas), dentre outras. Essas técnicas e métodos permitem aumentar o grau de racionalidade da decisão, auxiliando na definição das ações a serem tomadas, pois permitem considerar vários aspectos relevantes do processo decisório.

O goal programming, uma das técnicas da pesquisa operacional, envolve soluções de problemas que contêm não somente uma função objetivo, mas também várias metas que se deseja atingir. Requer um procedimento de solução iterativa pela qual o tomador de decisão investiga uma variedade de soluções para encontrar a que seja mais satisfatória.

Diferentemente da programação linear, o goal programming não resolverá um problema apresentando somente uma solução ótima, mas sim, apresentará uma série de soluções variadas do problema para que o tomador de decisão possa encontrar/escolher aquela que melhor the atender.

O objetivo deste artigo, portanto, é demonstrar, a partir de um exemplo prático, como o gestor pode aplicar a técnica do goal programming na formulação de alternativas de um plano operacional, com o auxílio da ferramenta Microsoft Excel, tendo em vista as facilidades hoje encontradas após o surgimento e o aprimoramento das planilhas eletrônicas.

Este artigo visa contribuir para a maior utilização dos modelos matemáticos e estatísticos no cotidiano empresarial, buscando melhorar o processo de tomada de 
A utilização da Goal Programming no processo de tomada de decisão Flávia Zobóli Dalmácio, Dimitri Pinheiro de Sant’Anna, Luciene Laurett Rangel, Valcemiro Nossa

decisão. A utilização de modelos facilita a compreensão de estruturas e relações complexas, pois segrega os aspectos fundamentais relativos ao problema em foco.

Os aspectos conceituais mais relevantes do goal programming são tratados na contextualização teórica e na aplicação da técnica. Por meio de um exemplo prático, demonstra-se a operacionalização dessa técnica, com o uso do recurso "solver", disponível na planilha eletrônica Microsoft Excel.

\section{CONTEXTUALIZAÇÃO}

\subsection{Breve Histórico}

Em novembro de 1961, uma sessão da American Association of Advertising Agencies foi aberta para uma exposição e análise do primeiro modelo de programação linear, que foi explicitamente preparado e divulgado para uso operacional e para implementação prática na área de planejamento e gestão (CHARNES et al., 1968, p. B423).

O desenvolvimento inicial do conceito de goal programming é atribuído a Charnes e Cooper, a partir dessa discussão em 1961, embora eles argumentem que a idéia originou-se, realmente, em 1952. Em essência, eles propuseram um modelo para lidar com certos tipos de problemas que envolviam programação linear e cujas metas eram incluídas como restrições. Já que era impossível satisfazer todas as metas, procuraram minimizar a soma dos valores absolutos dos desvios em relação a tais metas (IGNIZIO, 1978, p. 1109).

À publicação do livro de Charnes e Cooper, Management Models and Industrial Applications of Linear Programming (1961), seguiu-se a publicação de Yuji ljiri, Management Goals and Accounting for Control, em 1965, que refinava e reforçava a noção geral de goal programming. Como resultado destes esforços, o goal programming tornou-se um modelo de programação matemática operacional (KILLOUGH e SOUDERS, 1973, p. 269).

Outros pesquisadores exploraram as aplicações do goal programming, em áreas como: a) planejamento da produção e mão de obra; b) orçamento de capital; c) 
administração hospitalar; d) alocação de recursos acadêmicos; e) planejamento econômico municipal; f) problemas de transporte; g) recursos hidro-energéticos; h) projetos de sistemas de radar e sonar; i) seleção de portifólio; j) determinação de tempos padrões; I) estratégias de fusões, vôos de foguetes; m) resfriamento e aquecimento solar; n) determinação do nível de manutenção; o) modelos de demanda de carvão, dentre outras. Algumas destas aplicações não se limitam somente ao linear goal programming, mas utilizam modelos não lineares (nonlinear goal programming) ou lineares com variáveis discretas (linear integer goal programming) ou outros (IGNIZIO, 1978, p. 1112).

\subsection{Goal Programming}

Segundo Killough e Souders (1973, p. 270), goal programming, assim como a programação linear, é um modelo matemático linear, mas existem várias diferenças entre ambos. A técnica de programação linear limita-se a resolver problemas onde os objetivos da gerência podem ser estabelecidos em uma meta unidimensional, como a maximização de lucro ou minimização de custo. Porém, no dia-a-dia, a gerência se depara com muitas metas que, em geral, são incompatíveis e incomensuráveis, e transformar estas metas múltiplas em uma meta unidimensional, geralmente, não é possível. Felizmente, o goal programming apareceu para possibilitar a resolução de problemas de decisão com uma meta principal e múltiplas submetas, bem como, com múltiplas metas principais e múltiplas submetas.

Ragsdale (2001, p. 298) afirma que a maior parte das técnicas de programação linear assume que as restrições do modelo não podem ser violadas (hard constraints), entretanto, com o goal programming acontece justamente o inverso: as restrições do modelo são restrições, normalmente, "incompatíveis/contraditórias" entre si e, por isso, geralmente, viola-se pelo menos uma das restrições para se alcançar soluções possíveis. Estas restrições não tão rígidas (soft constraints) assemelham-se mais com "metas" desejáveis de se atingir, sem serem vistas como uma "camisa-de-força" pelo modelo. 
Em muitos casos reais o tomador de decisão depara-se com problemas cujo modelo de decisão está mais próximo do modelo do goal programming do que da programação linear, ou seja, não se tem apenas uma função objetivo a ser maximizada ou minimizada, mas sim, um conjunto de opções resultante de diversas restrições não totalmente compatíveis entre si. Normalmente, o tomador encara várias soluções onde "se ganha de um lado e se perde de outro". Estes problemas podem ser trabalhados com o goal programming.

Em geral, o goal programming apresenta os seguintes componentes: a) variáveis de decisão (variáveis reais que o modelo tenta otimizar); b) as restrições (conjunto de relacionamentos que restringem as variáveis de decisão); c) as variáveis de desvio (desvios positivos e negativos das variáveis de decisão em relação às metas); e d) a função objetivo (função que reflete o critério de otimização das variáveis). Tais componentes são melhores entendidos por meio de um exemplo prático.

\section{APLICAÇÃO PRÁTICA}

Para demonstrar os conceitos e uma melhor utilização da técnica do goal programming, por meio do recurso "solver" disponível na planilha eletrônica Microsoft Excel, apresenta-se uma aplicação prática, baseada em um exemplo hipotético. As etapas descritas, a seguir, são as mesmas propostas por Ragsdale (2001).

\subsection{Exemplo}

A empresa Delphi S.A. produz três tipos de produtos: camisa, bermuda e calça. Para fabricar camisa, a empresa consome 0,050 horas-máquinas (3,0 minutos) e gasta $\mathrm{R} \$ 20,00$ com custos e despesas variáveis. Para fabricar bermuda, a empresa consome 0,075 horas-máquinas ( 4,5 minutos) e gasta $R \$ 31,00$ com custos e despesas variáveis. Para fabricar calça, a empresa consome 0,125 horas-máquinas (7,5 minutos) e gasta $\mathrm{R} \$ 50,00$ com custos e despesas variáveis. Além disso, a empresa apurou que a margem de contribuição unitária da camisa é de $R \$ 4,00$; da bermuda é de $R \$ 6,00$; e da calça é de $R \$ 13,00$. A empresa tem uma disponibilidade mensal de até 2.520 horas- 
máquinas e $\mathrm{R} \$ 1.200 .000,00$ de capital para arcar com seus custos e despesas totais. Além disso, para cobrir os custos fixos $R \$ 200.000,00$ e obter um lucro de $R \$ 40.000,00$, a empresa precisa de, no mínimo, uma margem de contribuição total de $\mathrm{R} \$ 240.000,00$. De acordo com a carteira de pedidos de clientes, informada pela área de vendas, a empresa deve produzir, no mínimo, a quantidade de 13.000 camisas, 10.000 bermudas e 7.000 calças.

\subsection{Definição das Variáveis de Decisão}

Neste exemplo a decisão a ser tomada é: quantas camisas, bermudas e calças devem ser produzidas pela empresa? Estas quantidades são representadas pelas variáveis $\mathrm{X} 1, \mathrm{X} 2$ e X3, respectivamente.

\subsection{Definição de Metas (Goals)}

Diferentemente dos exemplos clássicos de programação linear, este problema não possui somente um objetivo específico, mas um conjunto de metas a serem alcançadas no mês, listadas abaixo:

- Meta 1 - produzir aproximadamente 13.000 camisas;

- Meta 2 - produzir aproximadamente 10.000 bermudas;

- Meta 3 - produzir aproximadamente 7.000 calças;

- Meta 4 - utilizar aproximadamente 2.520 horas-máquinas;

- Meta 5 - apresentar um custo total aproximado de $R \$ 1.200 .000,00$;

- Meta 6 - obter uma margem de contribuição total aproximada de $\mathrm{R} \$ 240.000,00$.

Nota-se que, a idéia de rigidez das metas (restrições) logo é descartada pela flexibilização proporcionada pela noção de aproximação, constante na lista acima, ou seja, estas "restrições" estão muito mais próximas de metas flexíveis do que de rígidas restrições. 
Se, por exemplo, as quatro primeiras metas puderem ser atingidas a um custo de $\mathrm{R} \$ 1.200 .050,00$, muito provavelmente, o desvio de $\mathrm{R} \$ 50,00$ não impedirá que esta solução seja considerada pelo tomador de decisão.

\subsection{Definição de Limitações das Metas}

O primeiro passo na formulação de um modelo para resolução de problemas por meio do goal programming, é criar limitações para cada uma das metas do problema. As limitações de metas permitem que se determine quão precisa deve ser a solução para cada meta.

Para entender como estas limitações devem ser formuladas, no exemplo são utilizadas as seguintes variáveis (restrições rígidas e flexíveis):

- Restrições Rígidas

$$
\begin{aligned}
& x_{1}=13.000 \text { (Camisa) } \\
& x_{2}=10.000 \text { (Bermuda) } \\
& x_{3}=7.000 \text { (Calça) } \\
& 4 x_{1}+6 x_{2}+13 x_{3}=240.000(\mathrm{MC}) \\
& 20 x_{1}+31 x_{2}+50 x_{3}+200.000=1.200 .000(\mathrm{CDF}+\mathrm{CDV}) \\
& 0,050 x_{1}+0,075 x_{2}+0,125 x_{3}=2.520(\mathrm{HMP})
\end{aligned}
$$

Onde:

$\mathrm{MC}=$ margem de contribuição

$\mathrm{CDF}=$ custos e despesas fixas

CDV = custos e despesas variáveis

HMP = horas-máquinas de produção

As equações apresentadas são restrições rígidas, mas se definidas na forma a seguir, permitem a flexibilidade de que o modelo do goal programming necessita. 
- Restrições Flexíveis

$$
\begin{aligned}
& x_{1}+d_{1}^{-}-d_{1}^{+}=13.000 \text { (Camisa) } \\
& x_{2}+d_{2}^{-}-d_{2}^{+}=10.000 \text { (Bermuda) } \\
& x_{3}+d_{3}^{-}-d_{3}^{+}=7.000 \text { (Calça) } \\
& 4 x_{1}+6 x_{2}+13 x_{3}+d_{4}^{-}-d_{4}^{+}=240.000(\mathrm{MC}) \\
& 20 x_{1}+31 x_{2}+50 x_{3}+200.000+d_{5}^{-}-d_{5}^{+}=1.200 .000(\mathrm{CDF}+\mathrm{CDV}) \\
& 0,050 x_{1}+0,075 x_{2}+0,125 x_{3}+d_{6}^{-}-d_{6}^{+}=2.520(\mathrm{HMP}) \\
& \text { Onde: } d_{i}^{-}, d_{i}^{+} \geq 0 \text { para todo i. }
\end{aligned}
$$

As variáveis $d_{i}^{-}$e $d_{i}^{+}$são chamadas variáveis de desvio. $0 d_{i}^{-}$representa o valor absoluto que cada meta está abaixo do originalmente desejado e o $d_{i}^{+}$representa o valor absoluto que cada meta está acima do originalmente desejado.

\subsection{Função Objetivo do Goal Programming}

Num problema modelado para o goal programming, o objetivo é determinar uma solução que atinja todas as metas, tão certeiramente quanto possível. A solução ideal, para qualquer problema de goal programming, seria aquela em que cada meta é atingida, exatamente, no ponto desejado (em tal solução ideal, todas as variáveis de desvio seriam iguais a zero). Geralmente, não é possível atingir a solução ideal, porque algumas metas são conflitantes entre si. Neste caso, procura-se uma solução que desvie o mínimo possível da solução ideal.

De acordo com Killough e Souders (1973, p. 270), o goal programming procura minimizar a soma dos desvios, contudo, como muitas vezes trabalha-se com variáveis que não podem ser somadas (horas, quantidade, valores monetários), o modelo transforma os desvios (números absolutos) em desvios percentuais em relação à meta desejada. Desta maneira, o modelo estaria tratando todos os desvios percentuais da mesma forma, o que, geralmente, não é desejável ao tomador de decisões. 
O goal programming, por isso, exige que o gestor atribua pesos (ponderações) aos desvios percentuais (privilegiando algumas metas em relação às outras), gerando, então, uma função objetivo final que visa minimizar a soma ponderada dos percentuais dos desvios:

$$
M I N: \sum_{i} \frac{1}{m_{i}}\left(p_{i}^{-} d_{i}^{-}+p_{i}^{+} d_{i}^{+}\right)
$$

Onde:

$m_{i}=$ meta para todo $\mathrm{i} ;$

$p_{i}^{-}=$peso relativo ao desvio para baixo para todo $\mathrm{i}$;

$p_{i}^{+}=$peso relativo ao desvio para cima para todo $\mathrm{i} ;$

$d_{i}^{-}=$desvio para baixo para todo i;

$d_{i}^{+}=$desvio para cima para todo i.

Para o tomador de decisão, a variável cujo desvio é considerado altamente indesejável apresenta um alto peso relativo (distante de zero), enquanto uma variável cujo desvio não seja tão indesejável apresenta um baixo peso relativo (próximo de zero). Caso haja um sentimento de neutralidade em relação ao desvio, o peso será nulo (igual a zero) (RENDER e STAIR, 1997, p. 532).

No entanto, não existe um procedimento padrão para o estabelecimento destes pesos, não garantindo que a primeira solução encontrada pelo modelo seja a solução mais desejável. Ou seja, o gestor deve, de maneira iterativa, estabelecer um conjunto de pesos para resolver o problema, analisar a solução e, então, redefinir os pesos e resolver o problema novamente. Pode ser necessário repetir este processo muitas vezes, até que o gestor esteja satisfeito com a solução encontrada. 
A utilização da Goal Programming no processo de tomada de decisão Flávia Zobóli Dalmácio, Dimitri Pinheiro de Sant'Anna, Luciene Laurett Rangel, Valcemiro Nossa

\subsection{Definição do Objetivo}

No exemplo dado, assume-se que o tomador de decisão considera indesejável o subatendimento das quatro primeiras metas, relacionadas às quantidades de produtos e à margem de contribuição, sendo para ele indiferente ou mesmo desejável a superação destas metas. Por outro lado, considera indesejável a superação das duas últimas metas, relacionadas aos custos e despesas totais e às horas-máquinas de produção, sendo para ele indiferente ou mesmo desejável, o subatendimento destas metas.

Neste caso, para minimizar a soma ponderada dos percentuais dos desvios, deve-se utilizar a seguinte função objetivo:

$M I N:\left(\begin{array}{l}\frac{p_{1}^{-}}{13.000} d_{1}^{-}+\frac{p_{1}^{+}}{13.000} d_{1}^{+}+\frac{p_{2}^{-}}{10.000} d_{2}^{-}+\frac{p_{2}^{+}}{10.000} d_{2}^{+}+\frac{p_{3}^{-}}{7.000} d_{3}^{-}+\frac{p_{3}^{+}}{7.000} d_{3}^{+}+ \\ +\frac{p_{4}^{-}}{240.000} d_{4}^{-}+\frac{p_{4}^{+}}{240.000} d_{4}^{+}+\frac{p_{5}^{-}}{1.200 .000} d_{5}^{-}+\frac{p_{5}^{+}}{1.200 .000} d_{5}^{+}+\frac{p_{6}^{-}}{2.520} d_{6}^{-}+\frac{p_{6}^{+}}{2.520} d_{6}^{+}\end{array}\right)$

No exemplo desenvolvido, é atribuído peso 1 abaixo $\left(p_{1}^{-}=p_{2}^{-}=p_{3}^{-}=1\right)$ e peso 0 acima $\left(p_{1}^{+}=p_{2}^{+}=p_{3}^{+}=0\right)$ para as quantidades de camisa, bermuda e calça; peso 4 abaixo $\left(p_{4}^{-}=4\right)$ e peso 0 acima $\left(p_{4}^{+}=0\right)$ para a margem de contribuição total; peso 2 acima $\left(p_{5}^{+}=2\right)$ e peso 0 abaixo $\left(p_{5}^{-}=0\right)$ para custos e despesas totais; e peso 5 acima $\left(p_{6}^{+}=5\right)$ e peso 0 abaixo $\left(p_{6}^{-}=0\right)$ para horas-máquinas.

A atribuição dos pesos (para cima ou para baixo), feita pelo gestor, indica penalidade, de acordo com a priorização das metas estabelecidas.

Vale notar que a função objetivo, apresentada na implementação do modelo a seguir, omite as variáveis cujo peso atribuído pelo tomador de decisão demonstre indiferença em relação ao desvio, ou seja, igual a zero. Neste exemplo, os desvios para cima, das quatro primeiras metas, não estão sendo penalizados e os desvios para baixo, das duas últimas metas, também não. 


\subsection{Implementação do Modelo}

O modelo de programação linear, deste exemplo, de goal programming é:

$M I N: \frac{1}{13.000} d_{1}^{-}+\frac{1}{10.000} d_{2}^{-}+\frac{1}{7.000} d_{3}^{-}+\frac{4}{240.000} d_{4}^{-}+\frac{2}{1.200 .000} d_{5}^{+}+\frac{5}{2.520} d_{6}^{+}$

Sujeito a:

$$
\begin{aligned}
& x_{1}+d_{1}^{-}-d_{1}^{+}=13.000 \text { (Camisa) } \\
& x_{2}+d_{2}^{-}-d_{2}^{+}=10.000 \text { (Bermuda) } \\
& x_{3}+d_{3}^{-}-d_{3}^{+}=7.000 \text { (Calça) } \\
& 4 x_{1}+6 x_{2}+13 x_{3}+d_{4}^{-}-d_{4}^{+}=240.000(\mathrm{MC}) \\
& 20 x_{1}+31 x_{2}+50 x_{3}+200.000+d_{5}^{-}-d_{5}^{+}=1.200 .000(\mathrm{CDF}+\mathrm{CDV}) \\
& 0,050 x_{1}+0,075 x_{2}+0,125 x_{3}+d_{6}^{-}-d_{6}^{+}=2.520(\mathrm{HMP})
\end{aligned}
$$

Onde:

$d_{i}^{-}, d_{i}^{+} \geq 0$ para todo $\mathrm{i}$

$x_{i} \geq 0$ para todo $\mathrm{i}$;

$x_{i}$ deve ser inteiro.

Nas equações demonstradas, os desvios têm o papel de ajustar o valor real encontrado ao valor desejado (alvo), ou seja, se o valor real gerado pelo modelo for menor que o desejado, soma-se o desvio para baixo $\left(d_{i}^{-}\right)$, se o valor real for maior que o desejado, diminui-se o desvio para cima $\left({ }^{d_{i}^{+}}\right)$.

Este modelo pode ser implementado em uma planilha Microsoft Excel e resolvido com o recurso "solver" do software, como desenvolvido a seguir.

Conforme ilustrado pela Figura 1, a primeira parte da planilha apresenta os dados básicos do problema, para que sejam efetuados os demais cálculos necessários 
ao modelo. A segunda parte apresenta as variáveis de decisão, as variáveis de desvio e as limitações às metas do problema. A terceira parte calcula os desvios relativos, a partir dos desvios absolutos apurados na segunda parte. A quarta parte recebe os pesos atribuídos pelo tomador de decisão aos desvios (para cima e para baixo), em relação a cada meta. E, a última parte apresenta, neste exemplo, o resultado da minimização da função objetivo.

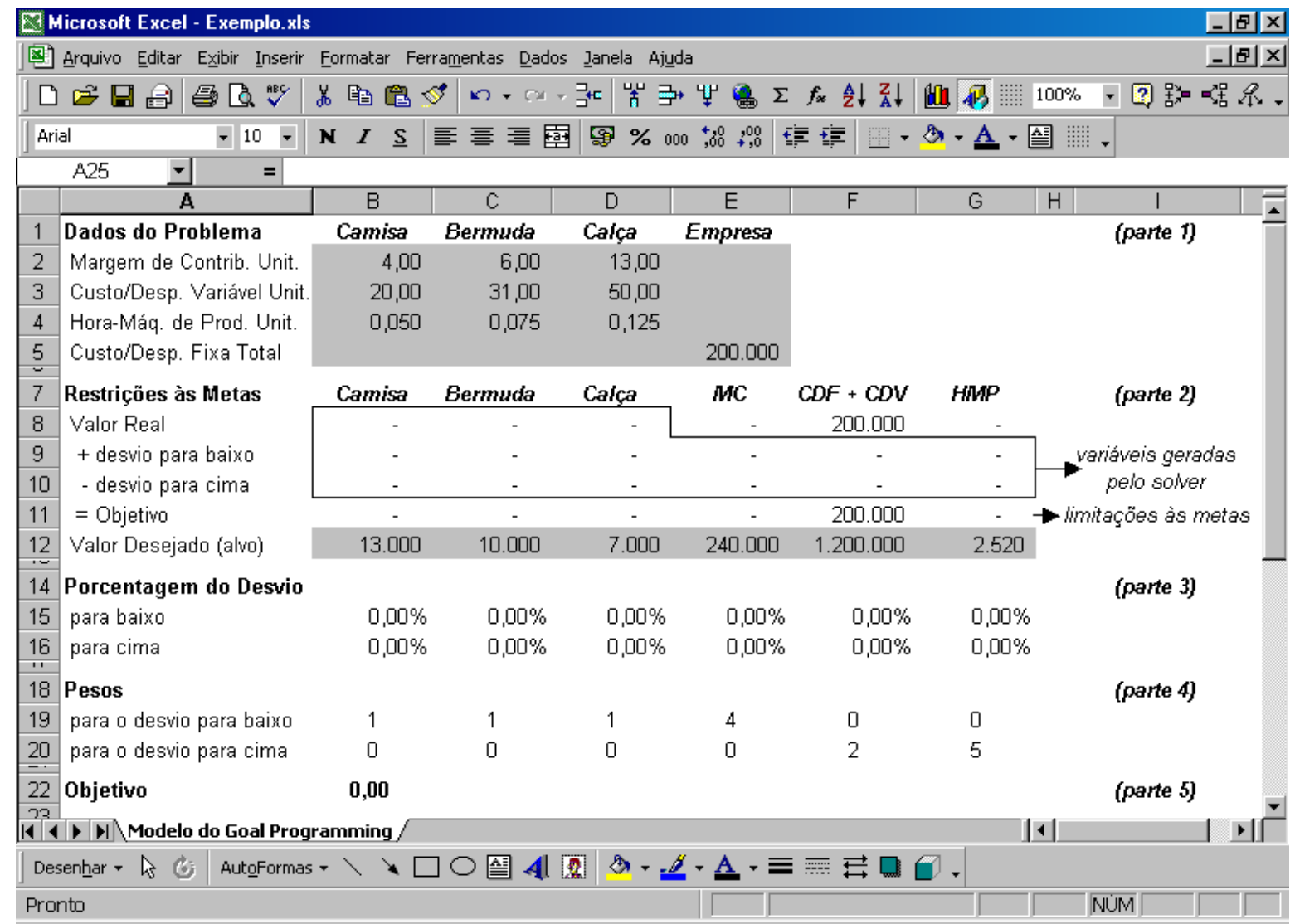

Figura 1: Modelagem do Goal Programming

$\mathrm{Na}$ Tabela 1, são descritas as fórmulas da Figura 1, necessárias para a elaboração da planilha eletrônica. 
A utilização da Goal Programming no processo de tomada de decisão

Flávia Zobóli Dalmácio, Dimitri Pinheiro de Sant’Anna, Luciene Laurett Rangel, Valcemiro Nossa

Tabela 1: Descrição das Fórmulas da Figura 1

\begin{tabular}{|c|c|c|}
\hline Variável do Modelo & Célula & Fórmula \\
\hline Quantidade da produção de camisas & B8 & $\left({ }^{*}\right)$ \\
\hline Quantidade da produção de bermudas & C8 & $\left({ }^{*}\right)$ \\
\hline Quantidade da produção de calças & D8 & $\left({ }^{*}\right)$ \\
\hline Margem de contribuição & E8 & =SOMARPRODUTO(B2:D2;B8:D8) \\
\hline Custos e despesas totais & F8 & =E5 + SOMARPRODUTO(B3:D3;B8:D8) \\
\hline Horas-máquinas de produção & G8 & =SOMARPRODUTO(B4:D4;B8:D8) \\
\hline Desvio para baixo de camisas & B9 & $\left(^{*}\right)$ \\
\hline Desvio para baixo de bermudas & C9 & $\left({ }^{*}\right)$ \\
\hline Desvio para baixo de calças & D9 & $\left({ }^{*}\right)$ \\
\hline Desvio para baixo de margem de contribuição & E9 & $\left({ }^{*}\right)$ \\
\hline Desvio para baixo de custos e despesas totais & F9 & $\left({ }^{*}\right)$ \\
\hline Desvio para baixo de horas-máquinas & G9 & $\left({ }^{*}\right)$ \\
\hline Desvio para cima de camisas & B10 & $\left({ }^{*}\right)$ \\
\hline Desvio para cima de bermudas & C10 & $\left({ }^{*}\right)$ \\
\hline Desvio para cima de calças & D10 & $\left({ }^{*}\right)$ \\
\hline Desvio para cima de margem de contribuição & E10 & $\left({ }^{*}\right)$ \\
\hline Desvio para cima de custos e despesas totais & F10 & $\left({ }^{*}\right)$ \\
\hline Desvio para cima de horas-máquinas & G10 & $\left({ }^{*}\right)$ \\
\hline Limitação à meta de camisas & B11 & $=\mathrm{B} 8+\mathrm{B} 9-\mathrm{B} 10$ \\
\hline Limitação à meta de bermudas & C11 & $=\mathrm{C} 8+\mathrm{C} 9-\mathrm{C} 10$ \\
\hline Limitação à meta de calças & D11 & $=\mathrm{D} 8+\mathrm{D} 9-\mathrm{D} 10$ \\
\hline Limitação à meta de MC & E11 & $=\mathrm{E} 8+\mathrm{E} 9-\mathrm{E} 10$ \\
\hline Limitação à meta de custos e despesas totais & $\mathrm{F} 11$ & $=\mathrm{F} 8+\mathrm{F} 9-\mathrm{F} 10$ \\
\hline Limitação à meta de horas-máquinas & G11 & $=\mathrm{G} 8+\mathrm{G} 9-\mathrm{G} 10$ \\
\hline Valor desejado de camisas & B12 & $\left({ }^{* *}\right)$ \\
\hline Valor desejado de bermudas & C12 & $\left({ }^{* *}\right)$ \\
\hline Valor desejado de calças & D12 & $\left({ }^{* \star}\right)$ \\
\hline Valor desejado de margem de contribuição & E12 & $\left({ }^{* *}\right)$ \\
\hline Valor desejado de custos e despesas totais & F12 & $\left({ }^{* \star}\right)$ \\
\hline Valor desejado de horas-máquinas & G12 & $\left({ }^{* *}\right)$ \\
\hline \% desvio para baixo (meta camisas) & B15 & $=\mathrm{B} 9 / \mathrm{B} \$ 12$ \\
\hline \% desvio para baixo (meta bermudas) & C15 & $=\mathrm{C} 9 / \mathrm{C} \$ 12$ \\
\hline \% desvio para baixo (meta calças) & D15 & $=\mathrm{D} 9 / \mathrm{D} \$ 12$ \\
\hline$\%$ desvio para baixo (meta margem de contrib.) & E15 & $=\mathrm{E} 9 / \mathrm{E} \$ 12$ \\
\hline \% desvio para baixo (meta custos/desp. totais) & F15 & $=\mathrm{F} 9 / \mathrm{F} \$ 12$ \\
\hline \% desvio para baixo (meta horas-máquinas) & G15 & $=\mathrm{G} 9 / \mathrm{G} \$ 12$ \\
\hline$\%$ desvio para cima (meta camisas) & B16 & $=\mathrm{B} 10 / \mathrm{B} \$ 12$ \\
\hline \% desvio para cima (meta bermudas) & C16 & $=\mathrm{C} 10 / \mathrm{C} \$ 12$ \\
\hline \% desvio para cima (meta calças) & D16 & $=\mathrm{D} 10 / \mathrm{D} \$ 12$ \\
\hline \% desvio para cima (meta margem de contrib.) & E16 & $=\mathrm{E} 10 / \mathrm{E} \$ 12$ \\
\hline \% desvio para cima (meta custos/desp. totais) & F16 & $=\mathrm{F} 10 / \mathrm{F} \$ 12$ \\
\hline \% desvio para cima (meta horas-máquinas) & G16 & $=\mathrm{G} 10 / \mathrm{G} \$ 12$ \\
\hline Peso do desvio para baixo (meta camisas) & B19 & $\left({ }^{* *}\right)$ \\
\hline Peso do desvio para baixo (meta bermudas) & C19 & $\left({ }^{* *}\right)$ \\
\hline
\end{tabular}


A utilização da Goal Programming no processo de tomada de decisão Flávia Zobóli Dalmácio, Dimitri Pinheiro de Sant'Anna, Luciene Laurett Rangel, Valcemiro Nossa

\begin{tabular}{|c|c|c|}
\hline Peso do desvio para baixo (meta calças) & D19 & $\left({ }^{* \star}\right)$ \\
\hline Peso do desvio para baixo (meta MC) & E19 & $\left({ }^{* *}\right)$ \\
\hline Peso do desvio para baixo (meta custos totais) & F19 & $\left({ }^{* \star}\right)$ \\
\hline Peso do desvio para baixo (meta horas-máq.) & G19 & $\left({ }^{* *}\right)$ \\
\hline Peso do desvio para cima (meta camisas) & B20 & $\left({ }^{* *}\right)$ \\
\hline Peso do desvio para cima (meta bermudas) & C20 & $\left({ }^{* \star}\right)$ \\
\hline Peso do desvio para cima (meta calças) & $\mathrm{D} 20$ & $\left({ }^{* *}\right)$ \\
\hline Peso do desvio para cima (meta MC) & E20 & $(* \star)$ \\
\hline Peso do desvio para cima (meta custos totais) & F20 & $\left({ }^{* \star}\right)$ \\
\hline Peso do desvio para cima (meta horas-máq.) & G20 & $(* *)$ \\
\hline Objetivo & B22 & $=$ =SOMARPRODUTO(B15:G16;B19:G20) \\
\hline
\end{tabular}

Fonte: Elaborada pelos autores.

\subsection{Solução do Modelo}

O modelo pode ser resolvido, utilizando o recurso "solver", conforme ilustrado pelas Figuras 2, 3, 4 e 5.

Para acessar o "solver", clicar na opção "ferramentas", disponível na barra de ferramentas da planilha eletrônica Microsoft Excel e, então, preencher os seguintes parâmetros:

- Definir célula destino: indicar a célula onde será exibido o resultado final da função objetivo;

- Igual a: identificar o tipo de solução que se deseja obter para o problema (Máx = maximizar; Mín = minimizar; Valor de = indicar um valor específico);

- Células variáveis: indicar o endereço das células variáveis da planilha;

- Submeter às restrições: clicar sobre o botão adicionar, informar as células que possuem restrições, escolher na lista de opções o tipo de restrição desejada e indicar a restrição. 


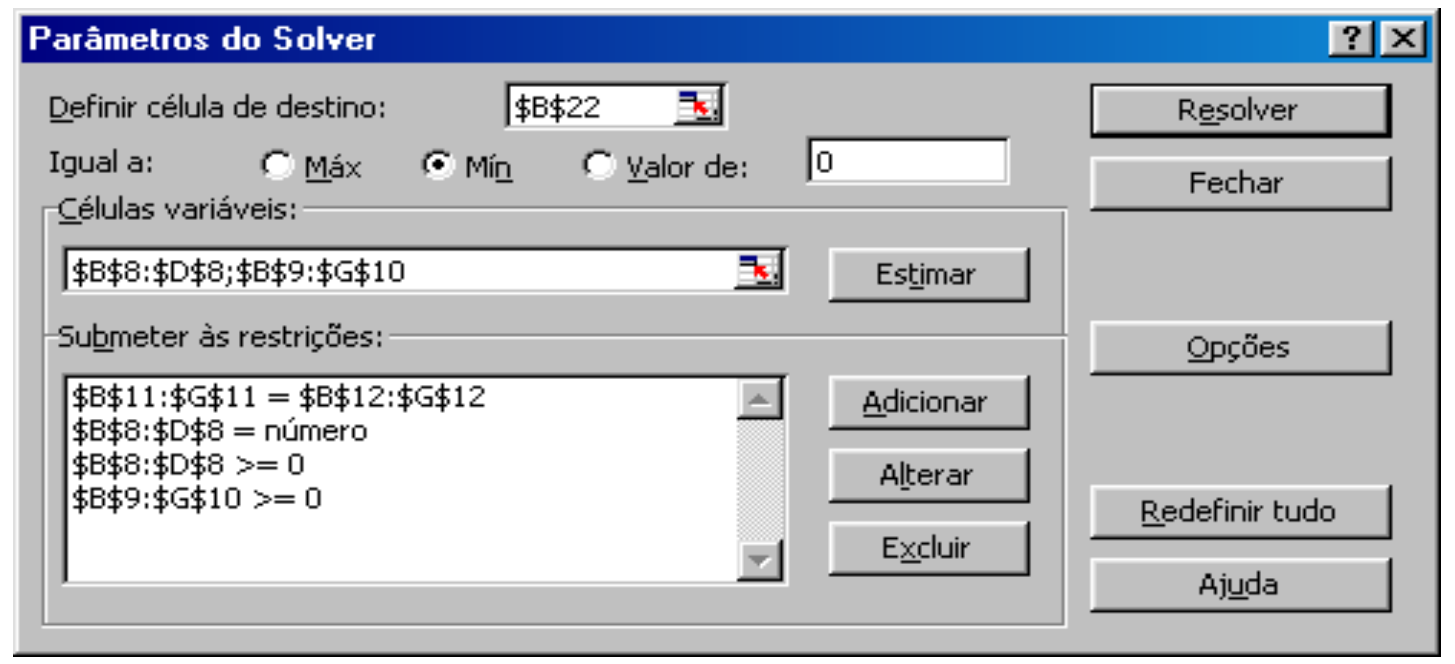

Figura 2: Parâmetros do Solver

Definidos os parâmetros, o passo seguinte é definir as opções do "solver", clicando no botão "opções".

Nesta fase são indicadas as opções que permitem administrar a forma como o programa irá resolver o problema. Essas opções possibilitam a melhoria da precisão do resultado obtido e o tempo consumido na solução do problema. Dependendo da escolha, a solução poderá ser encontrada com maior ou menor rapidez, com maior ou menor precisão. No entanto, o "solver" apresenta uma pré-definição padronizada, devendo ser alterada somente em situações de resoluções mais complexas. 


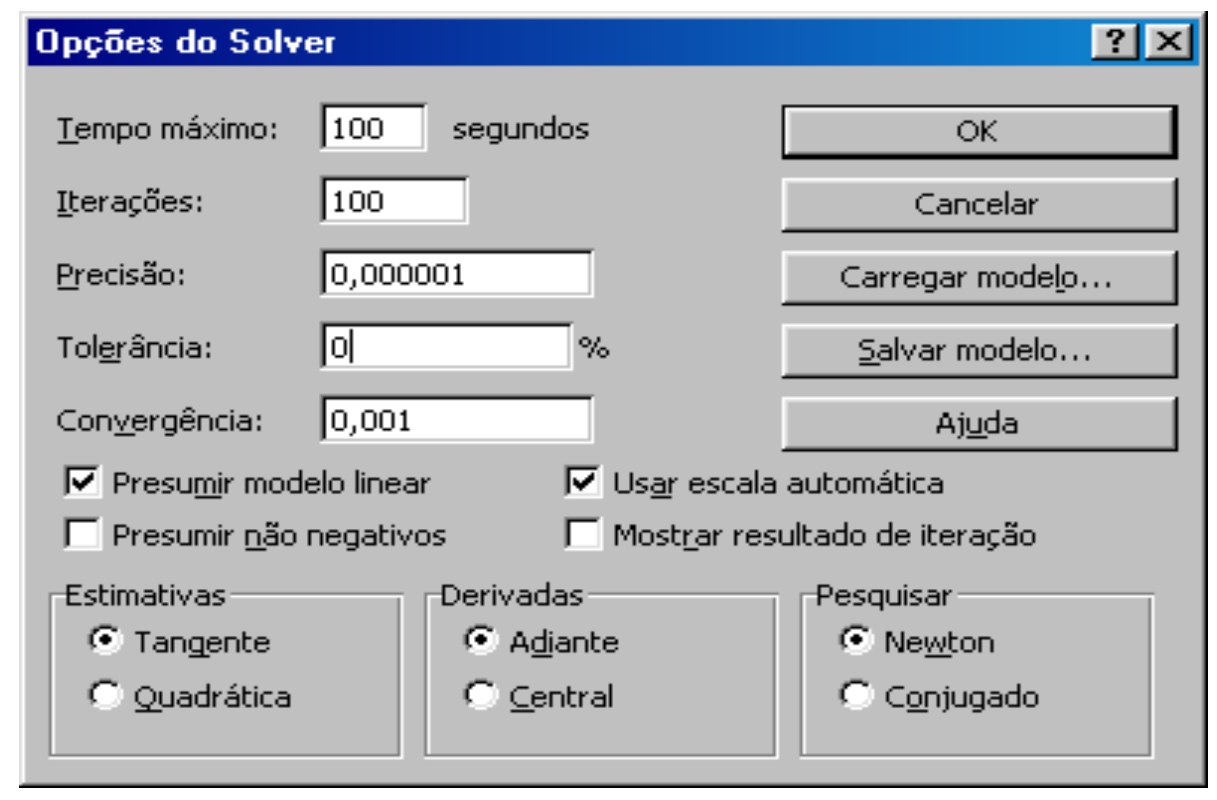

Figura 3: Opções do Solver

Após certificar-se de que todos os dados estão preenchidos corretamente, clicar em "ok" e, em seguida, "resolver".

Quando a solução para o modelo for encontrada, aparecerá a janela de resultados do solver. Pode-se, então, "salvar" a solução, retornar aos valores originais, ou então, solicitar a geração dos relatórios (relatório de resposta, relatório de sensibilidade e relatório de limites), conforme as opções oferecidas.

Neste exemplo, não é necessária a geração destes relatórios, basta, somente, clicar no botão "ok" e analisar os resultados.

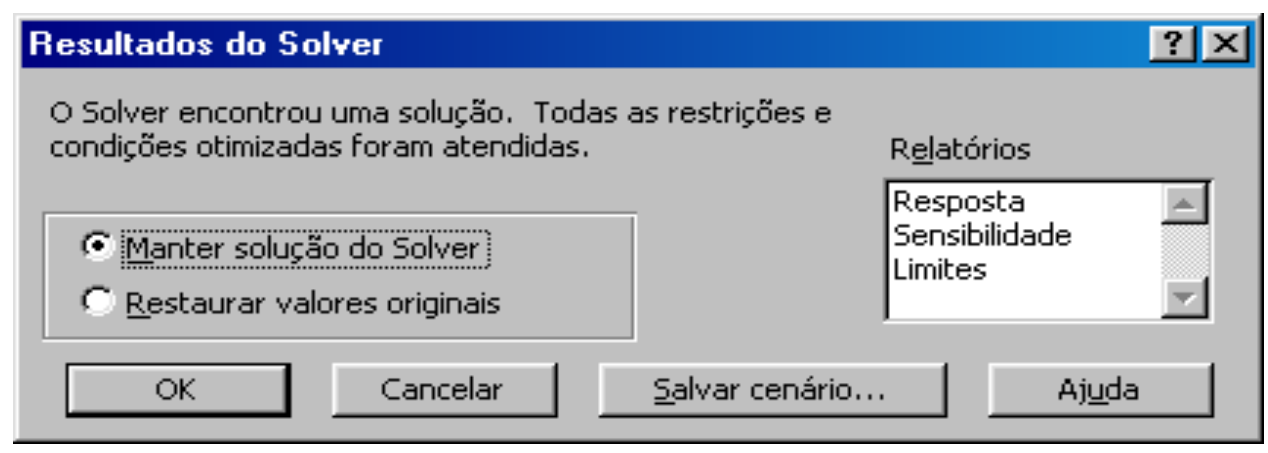

Figura 4: Resolução do Solver 
Na planilha final, ilustrada na Figura 5, são indicados: a quantidade de camisas, bermudas, calças, margem de contribuição total, custos e despesas totais e horasmáquinas de produção, necessários para atender o modelo.

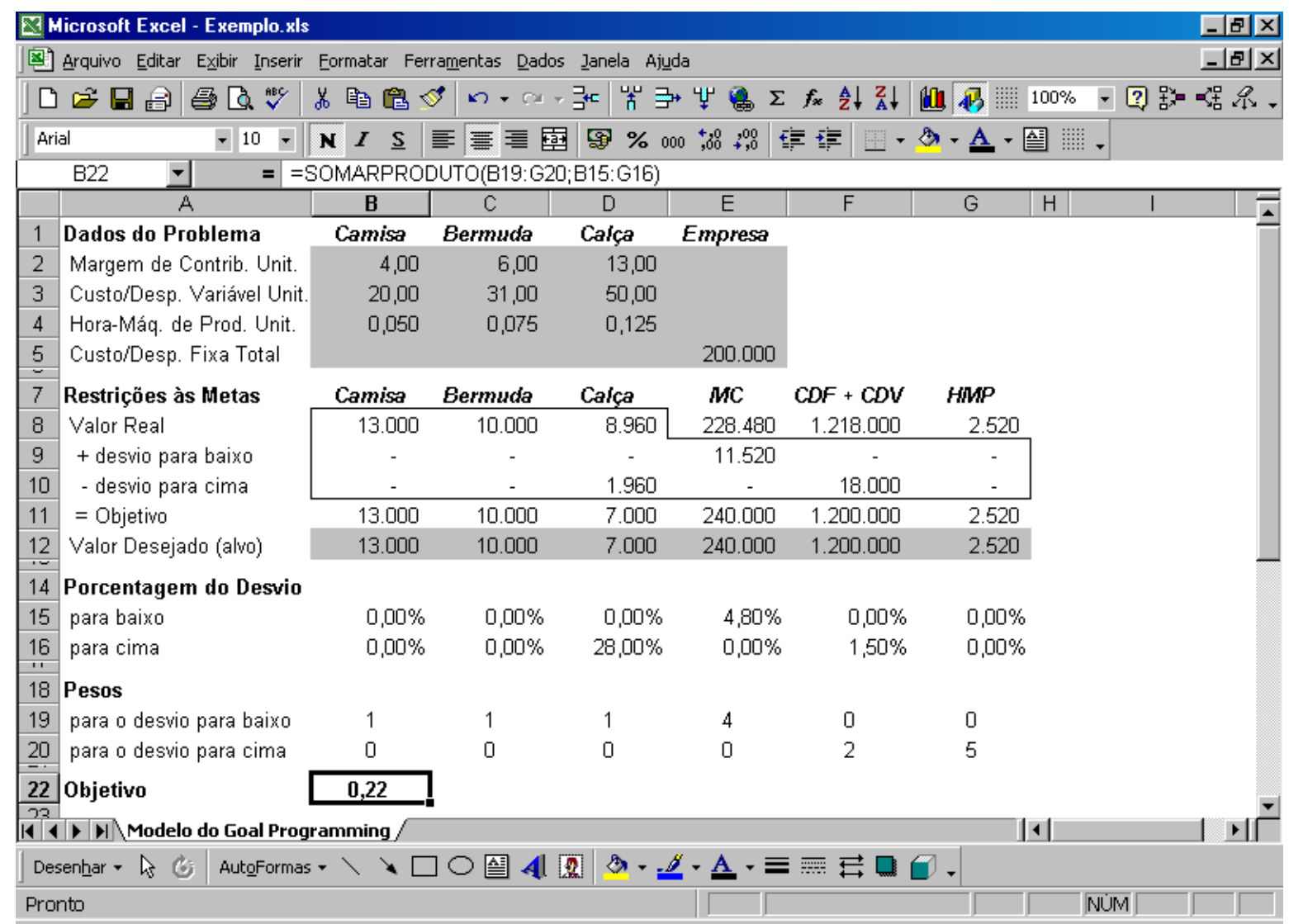

Figura 5: Primeira Solução do Modelo

\subsection{Análise da Solução}

Conforme observado na Figura 5, esta solução indica que a empresa deve produzir 13.000 camisas, 10.000 bermudas e 8.960 calças. Com isso, pode-se notar que, as metas de quantidade a produzir de camisas, bermudas e calças (a quantidade de calças, inclusive, superou a meta prevista em $28,0 \%$, de forma desejável) e a meta de horas-máquinas utilizada na produção foram atingidas. Entretanto, com as variáveis consideradas no modelo, não foi possível, nesta primeira solução, atingir a meta de 
margem de contribuição total (ficando abaixo da meta prevista em 4,8\%) e a meta do valor de custos e despesas totais (ficando acima da meta prevista em 1,5\%).

O valor objetivo de 0,22 foi gerado pela minimização da soma ponderada dos percentuais dos desvios $(0,22=28,0 \% \times 0+4,8 \% \times 4+1,5 \% \times 2)$. A superação da meta de produção de calças em $28,0 \%$ não afetou o resultado de 0,22 , já que esta superação não é indesejada pelo tomador de decisão, por isso, ele não atribuiu peso a este desvio para cima.

\subsection{Revisão do Modelo}

A primeira solução encontrada pode satisfazer o gestor, no entanto, como na elaboração do planejamento é comum a simulação de várias alternativas para a escolha da melhor, é bem provável que ele queira testar outras possíveis soluções.

Por exemplo, havendo demanda para esta quantidade de calças (8.960), que suporte um aumento na margem de contribuição unitária em $R \$ 2,00$ (o que implicaria num aumento no preço do produto), o gestor poderia, então, alterar a margem de contribuição unitária da calça de $R \$ 13,00$ para $R \$ 15,00$ e verificar a nova solução encontrada pelo modelo, conforme ilustrado pela Figura 6. 


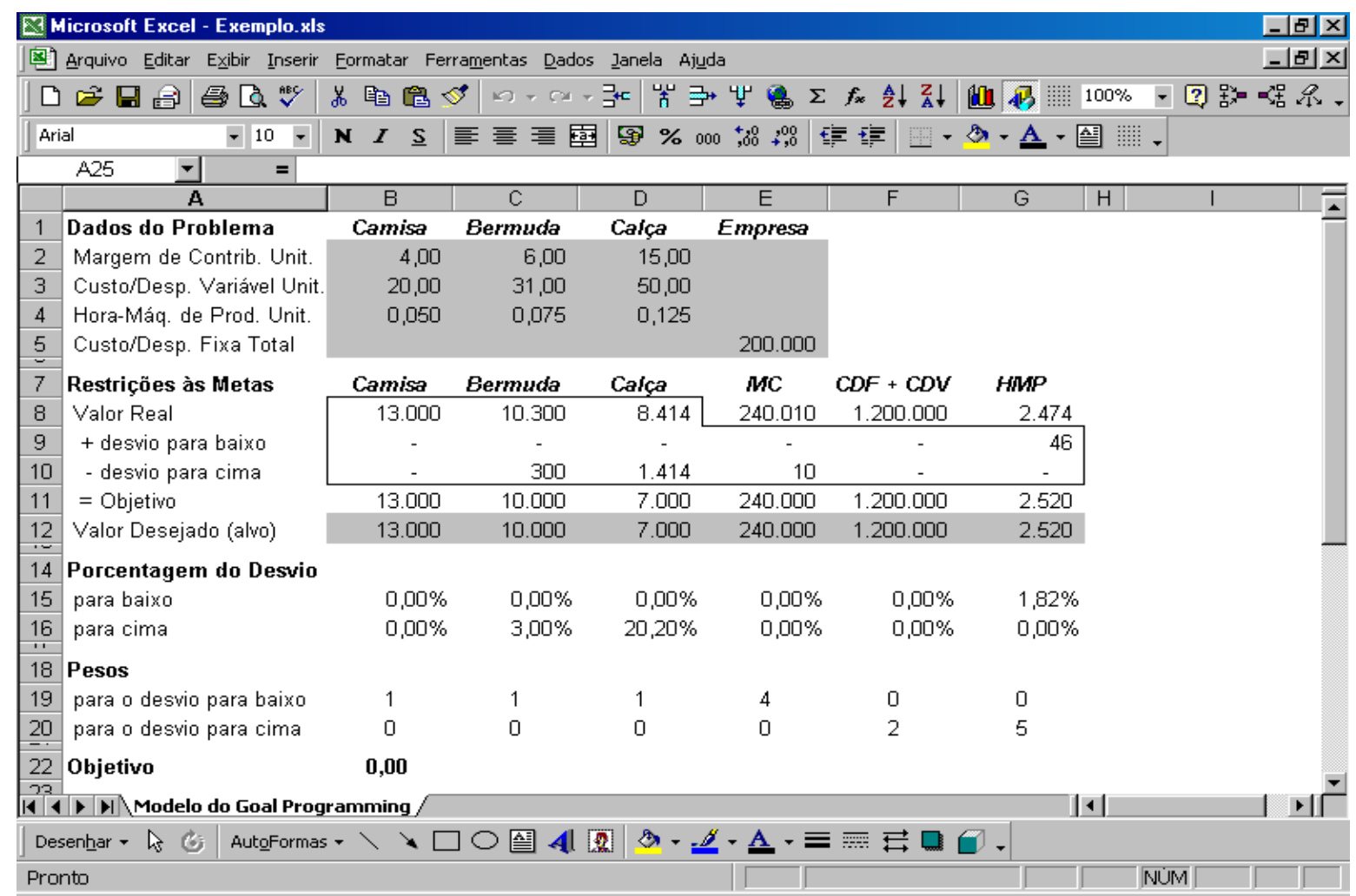

Figura 6: Outra Solução do Modelo

Nesta solução alternativa, observa-se que todas as metas foram atingidas com sucesso e/ou apresentaram desvios favoráveis à empresa, o que levou a minimização da soma ponderada dos percentuais dos desvios a atingir um valor objetivo igual a zero.

Por outro lado, seria relevante para o processo decisório, que fossem analisadas outras alternativas como, por exemplo: o mercado poderia não aceitar este aumento de preço e a empresa tentaria reduzir seus custos e despesas fixas totais. Neste caso, mantendo-se a margem de contribuição em $R \$ 13,00$, reduzindo-se o custo fixo total em $\mathrm{R} \$ 11.520,00$ (valor do desvio para baixo da margem de contribuição total apurado na primeira solução do modelo) e mantendo-se todas as outras condições constantes, chegar-se-ia ao valor objetivo de 0,20 ("melhor" do que o valor objetivo de 0,22 da primeira solução). Mesmo que os custos e as despesas totais atinjam valores indesejáveis (ainda que menores do que na primeira solução) e a margem de 
A utilização da Goal Programming no processo de tomada de decisão Flávia Zobóli Dalmácio, Dimitri Pinheiro de Sant’Anna, Luciene Laurett Rangel, Valcemiro Nossa

contribuição fique igual à primeira solução $(R \$ 228.480,00)$, a empresa, ainda assim, teria um lucro de $\mathrm{R} \$ 40.000,00$.

\section{CONSIDERAÇÕES FINAIS}

Neste artigo demonstrou-se, com a utilização de um exemplo prático, como o gestor pode aplicar a técnica do goal programming na formulação de alternativas de um plano operacional, melhorando o processo decisório com a geração e análise de diversos cenários.

O goal programming, diferentemente da programação linear, envolve soluções de problemas que contêm não somente uma função objetivo, mas sim várias metas que se deseja atingir. Esta técnica requer um procedimento de solução iterativa pela qual o tomador de decisão investiga uma variedade de soluções, para encontrar uma que seja mais satisfatória. Mais importante que o valor objetivo, encontrado ao final de cada iteração é, exatamente, a exploração de inúmeras possíveis soluções. Porém, alguns autores criticam o modelo do goal programming, por este não determinar somente um ponto ótimo, entendendo isso como uma limitação.

O goal programming é uma técnica que pode ser muito mais utilizada pelos gestores, no dia-a-dia das empresas, já que o modelo é aplicável em um software, de fácil acesso e manuseio, como o Microsoft Excel.

Apesar de neste trabalho não terem sido realizadas simulações com mudanças dos pesos (ponderações), referentes aos diversos tipos de variáveis de decisão (e seus desvios), esta possibilidade é uma das outras vantagens que pode ser explorada pelos gestores nas empresas.

O goal programming, por meio de simulações e modelos, permite que os gestores, durante o processo de planejamento ou de reestruturação da empresa, possam estar, continuamente, revendo suas prioridades e a própria hierarquia das metas/objetivos da empresa. 
A utilização da Goal Programming no processo de tomada de decisão Flávia Zobóli Dalmácio, Dimitri Pinheiro de Sant'Anna, Luciene Laurett Rangel, Valcemiro Nossa

\section{REFERÊNCIAS}

ANDRADE, E. L. (2000). Introdução à pesquisa operacional: métodos e modelos para a análise de decisão. (2 ed.). Rio de Janeiro, LTC, 277 p.

ARANTES, N. (1998). Sistemas de gestão empresarial: conceitos permanentes na administração de empresas válidas. (2 ed.). São Paulo, Atlas, 439 p.

CHARNES, A. et al. (1968). A goal programming model for media planning. Management Science. Disponível em: http://www.jstor.org/. Acesso em: 15/junho/2003.

IGNIZIO, J. P. (1978). A review of goal programming: a tool for multioobjective analysis. The Journal of the Operation Research Society. Disponível em: http://www.jstor.org/. Acesso em: 15/jun/2003.

KILLOUGH, L. N. e SOUDERS, T. L. (1873). A goal programming model for public accounting firms. The Accounting Review. Disponível em: http://www.jstor.org/. Acesso em: 15/junho/2003.

RAGSDALE, C. T. (2001). Spreadsheet modeling and decision analysis. (3 ed.). Cincinnati, Ohio, South-Western, 794 p.

RENDER, B. e RALPH M. (1997). Quantitative analysis for management. (6 ed.). New Jersey, Prentice Hall, $840 \mathrm{p}$.

Data de Submissão: 31/05/2008

Data de Aceite: 21/08/2008 